Zeitschrift für Augenheilkunde 1934;82:I-IV

\title{
Contents, Vol. 82, 1934
}

Inhaltsverzeichnis.

Eigenarbeiten. Seite

Bistis, J., Neuer Beitrag über die Sympathikusheterochromie 180 Back, J., Untersuchungen des vorderen Bulbusabschnittes

mit dem Fluoreszenzmikroskop

Freyler, H., Erfahrungen mit dem Piezometer von Gutmann 127 Heine, L., Bemerkungen ,,Zur

Frage des Operationszwanges

bei Unfallgeschädigten” unter besonderer Berücksich-

tigung der Verletzungsfolgen am Auge 1

Jeß, A., Zur Frage der Augenhintergrundsbefunde bei Nacht-

blindheit 177

Jessel, E., Beitrag zur Klinik und Therapie der intraorbi-

talen Tumoren 31

John, J., Keratitis parenchymatosa und Sattelnase ... 12

Kassner, H., Über Knochenbildung im Auge 139

Killmann, Über eine seltene Form feinst punktierter Horn-

hautveränderungen (Cornea farinata) 123

Kyrieleis, W., Über Augenhintergrundsveränderungen bei

Blutkrankheiten

15

Lyon, E., Übereinen Fall von Tay-Sachs scher amaurotischer

Idiotie ohne Makulabefund 188

Malkin, B., Über orbitale Alkoholinjektionen

286

Mylius, K., Über das Meningiom der Olfaktoriusrinne, eine

für den Augenarzt besonders wichtige Geschwulst . . 257 Pillat, A., Über eine eigenartige senile

Entartung der Leder-

haut an den Ansatzstellen der geraden Augenmuskeln 113

Rauh, W., Erfahrungen mit Pantocain 134

Riechert, T,, Über eine Ergänzung zur Lochbrille nach Lindner 191

Uuäelt, J., Zur Pathogenese des Trachoms 277

Schtscheglowa, A. A., Über Orbitaltumoren 194

Zeitler, M., Zur Klinik der epibulbären Tumoren. (Hierzu

Tafel I) $\quad 3$

$-\mathrm{IV}-$

Aus der Praxis für die Praxis. Seite

Mieses-Rei†, W. M., Luxatio bulbi posttraumatica .... 211

Beriehte über die ophthalmologische Literatur.

Nystagmus. Von Dr. A. Kestenbaum in Wien

Aderhaut. Netzhaut. Von Privatdozent Dr. W. Riehm in Würz-

burg 73 
Sehbahn, Auge und Nervenkrankheiten. Von Privatdozent Werner Kyrieleis in Hamburg 141

Normale und pathologische Anatomie. Von Privatdozent Dr.

R. Schneider-Graz 213

Entwicklungsgeschichte des Auges. Mißbildungen. Anthropologie.

Von Prof. R. Seefelder-Innsbruck:

Entwicklungsgeschichte des Auges 226

Mißbildungen 230

Anthropologie 247

Geschichte der Augenheilkunde. Von Prof. Dr. R. Seefelder-ïnns-

bruck 248

Physiologische und psychologische Optik. Von Privatdozent Dr.

H. Gasteiger-Innsbruck 299

$<$ xesellschaftsberichte.

Ophthalmologische Gesellschaft in Wien, Sitzungvom 16. Okt. 1933

Sitzung vom 20. Nov. 1933322

Sitzung vom 11. Dez. 1933330

Ungarische Ophthalmologische Gesellschaft. XXV. Jahresver-

sammlung in Budapest am 9.-11. Juni 1933

337

Diagnose und Therapie 105, 172, 254, 348

Buchbesprechungen 109, 256, 353

Personalien 256, 353

S. Sachregister zu Band $82 \quad 354$

Namenregister zu Band $82 \quad 360$ 